\title{
Valutazione multidimensionale dei bisogni del paziente con sclerosi multipla: aspetti sanitari e socio-assistenziali
}

\section{Luigi Maria Grimaldi}

U.O. Neurologia, Fondazione Istituto "G. Giglio", Cefalù (PA)

\section{Introduzione}

La sclerosi multipla (SM) è la più frequente malattia invalidante del giovane adulto dopo gli incidenti stradali, ma è pur sempre una malattia relativamente poco frequente $(0,09 \%$ della popolazione generale) . I suoi costi sanitari, tuttavia, sono enormi proprio perché colpisce la popolazione lavorativamente più attiva, ha una durata di varie decine di anni e si avvale di alcuni dei farmaci più costosi che esistano con un notevole aggravio per i Sistemi Sanitari e le singole Aziende Sanitarie. La sua storia naturale è stata

\section{Indirizzo per la corrispondenza:}

Luigi Maria Grimaldi

e-mail: luigi.grimaldi@hsr.it

Accettato: 17/12/2015 - Pubblicato online: 23/12/2015

(C) 2015 The Authors. This article is published by HPS Srl and licensed under Creative Commons Attribution-NC-ND 4.0 International (CC BY-NC-ND 4.0). Any commercial use is not permitted and is subject to Publisher's permissions. Full information is available at www.aboutpharma.com/publishing/riviste/aboutopen/ significativamente modificata dall'avvento di numerosi farmaci immunomodulanti/immunosoppressori che sono in grado di allentare l'aggressione autoimmunitaria nei confronti delle strutture del sistema nervoso centrale (SNC) [principalmente mielina e assoni]. L'accesso a queste molecole deve sottostare a buone pratiche cliniche che ne regolano l'impatto sanitario ma sono ancora carenti in quest'ambito: le strategie di integrazione dei vari farmaci oggi presenti nell'armamentario terapeutico, le terapie per le forme progressive (sia primarie che secondarie), le terapie riparative delle lesioni strutturali determinate dai processi di demielinizzazione cerebrale, il trattamento di eventi indesiderati frequentemente riscontrati con le principali terapie specifiche per la SM (disease modifying therapy, DMT) con potenzialità anche fatali [leucoencefalopatia multifocale progressiva (PML), altre encefaliti non JCV-correlate, porpora trombocitopenica idiopatica (PTI) ecc.] e, infine, le terapie per le forme pediatriche.

Le problematiche socio-assistenziali costituiscono 
spesso il motivo di principale sofferenza personale di una notevole quota di pazienti affetti da SM. La creazione di Percorsi Diagnostico-Terapeutici Assistenziali (PDTA) regionali ha aggiornato alle realtà territoriali l'ampia rete italiana di Centri SM, inserendo varie tipologie di rete (HubESpoke, rete diffusa ecc.). L'integrazione tra centri SM e rete territoriale della cronicità è ancora in corso e dovrebbe migliorare la gestione dei pazienti con più problemi di autosufficienza mediante l'inserimento nel circuito delle cure domiciliari o, ove necessario, residenziali (RSA). Il supporto riabilitativo, psicologico ed economico ai pazienti e alle loro famiglie, pur auspicato e richiesto a gran voce anche dalle associazioni dei malati [in Italia tra tutte l'Associazione Italiana Sclerosi Multipla (AISM)], è minacciato dalle restrizioni economiche di tutti i Sistemi Sanitari occidentali e di fatto non disponibile per un'ampia quota dei malati italiani.

\section{Gli attuali bisogni sanitari dei pazienti}

La storia naturale della SM, come riportata sin dalla sua prima descrizione nel 1860 fatta da Charcot, non è molto cambiata fino all'inizio degli anni ' 90 . Due erano le forme principali di decorso di malattia, con la forma recidivante-remittente tipica dell' $85 \%$ circa dei pazienti tendente a virare verso una fase di secondaria progressione nella maggior parte di essi dopo 10-20 anni di decorso in corrispondenza dell'aggravarsi dello stato clinico. Da 20-25 anni, forse per le mutate condizioni di vita nel mondo occidentale (più igiene, diagnosi sempre più precoci, riduzione delle malattie infettive anche grazie ai vari vaccini), si è assistito a un progressivo "ammorbidimento" della SM con esordio sempre più precoce ma con minore attività infiammatoria cerebrale e conseguente riduzione del tasso annuale di ricaduta e della gravità di ogni singolo attacco.

Su questo background si sono inseriti i vari farmaci immunomodulanti registrati per la prevenzione di nuove ricadute della SM, che hanno probabilmente contribuito a rallentare sostanzialmente il decorso di malattia e, forse, anche a consentire un più accele- rato recupero dalla disabilità allentando l'aggressione autoimmunitaria nei confronti delle strutture del SNC (mielina e assoni principalmente). Interessante notare come la gran parte di questi farmaci esibisca meccanismi d'azione completamente diversi gli uni dagli altri, consentendo un ampio spettro di alternative terapeutiche "sensate" qualora le prime linee non esercitino alcun effetto clinico positivo. Un elenco in continuo aggiornamento dei farmaci attuali è riportato nella Figura 1.

Numerosi studi hanno dimostrato come l'efficacia di tali farmaci sia inversamente proporzionale al tempo intercorso tra la diagnosi e l'inizio di un trattamento con DMT: quanto più precoce è tale intervento tanto minori sono la disabilità, l'accumulo di lesioni cerebrali, il tasso annuale di ricadute, tendendo alla condizione di "nessuna evidenza di attività di malattia" (NEDA) [1] Nel caso non ancora risolto delle terapie per le forme pediatriche di SM, tale imperativo categorico è frenato proprio dalla mancanza di studi specifici per tale fascia di età, anche se vari trials (con dimetilfumarato, teriflunomide, fingolimod e natalizumab) sono in fase di completamento proprio per questi pazienti.

Un secondo punto di sviluppo futuro sarà l'uso di marcatori di attività di malattia per monitorarne l'andamento e la risposta ai DMT: il monitoraggio degli effetti mediante marcatori surrogati come i marcatori biologici, l'imaging strutturale (sia lesionale che mirato alla valutazione dell'atrofia globale) e quello funzionale (es. PET microgliale), la valutazione e gestione del danno cognitivo e l'uso di farmaci sintomatici per le principali problematiche di questi pazienti (urinarie, muscolari, cognitive ecc.).

Tra i più rilevanti "unmet needs" sanitari della SM si pongono le strategie terapeutiche, che sono state rivoluzionate dall'arrivo di numerosi farmaci con meccanismi d'azione non sovrapponibili, diversi profili di sicurezza e di efficacia ma incerta capacità di combinazione reciproca. Un primo problema è dato dalla scelta della strategia di inizio terapia (es. induction vs escalation). Contrariamente a quanto routinariamente applicato nella gran parte delle malattie autoimmunitarie dell'uomo (artrite reumatoide, tiroidite autoimmune, miastenia gravis, morbo di Crohn 


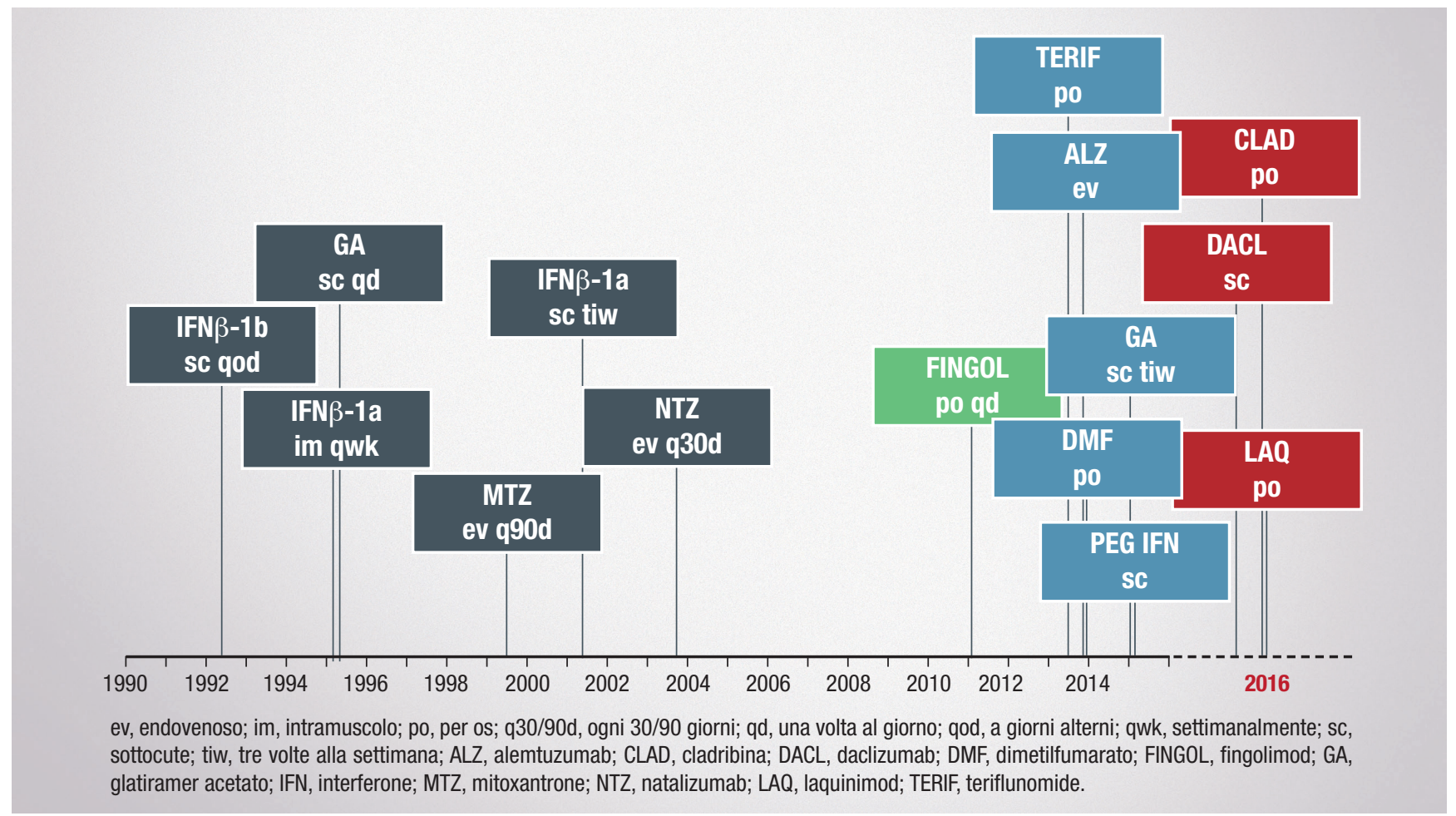

Figura 1. Presente e "futuro prossimo" delle terapie modificanti la malattia (DMT) per la sclerosi multipla.

ecc.), per i pazienti affetti da SM che iniziano per la prima volta un trattamento generalmente non viene previsto un bolo ad alte dosi per "bastonare" il sistema immunitario e riportarlo in condizioni di normo- se non ipo-funzione relative per poi proseguire con una terapia di mantenimento (sia a dosaggi più bassi che con altri farmaci meno potenti ma, magari, più maneggevoli e sicuri: induction therapy); si procede invece con un trattamento a basso impatto sia per efficacia percentuale che per effetti indesiderati per poi passare ad altri farmaci con efficacia progressivamente più alta, giustificandone la minore tollerabilità o i maggiori rischi con il precedente fallimento terapeutico (escalation therapy). Alla luce della ben nota "finestra terapeutica" dei primi mesi/anni post-diagnosi in cui è più probabile impedire la tendenza alla progressione della SM, e del noto aforisma anglosassone "time is brain" (il tempo perso a trovare una terapia efficace equivale a perdita di tessuto cerebrale...), sembrerebbe più logico applicare anche alla SM una terapia di induzione, possibilmente utilizzando quei farmaci che hanno la capacità di "resettare" il sistema immunitario come, ad esempio, alemtuzumab o ocrelizumab (Figura 2).

Per il primo di questi farmaci sono già stati dimostrati dati di efficacia confermata fino a 5 anni dal primo ciclo infusionale, che ne convalidano il potere di induzione [2] (Figura 3).

Un secondo punto critico sono le strategie d'integrazione dei vari farmaci (es. in combinazione, in successione seriale più o meno "ragionata" sulla base dei meccanismi di azione e degli effetti biologici conseguenti al loro uso): trials di fase II/III dovrebbero essere realizzati per definire queste importanti combinazioni, analogamente a quanto avvenuto tra gli anni '50 e '80 del secolo scorso per i farmaci antineoplastici, che oggi vengono praticamente sempre usati in combinazione o in modo seriato. È evidente che, invece, nel mondo della SM tale strada non è stata intrapresa durante lo sviluppo dei farmaci e appare improbabile che oggi siano possibili partnership tra differenti aziende farmaceutiche che hanno appena commercializzato i nuovi farmaci dopo vari lustri di (oneroso) sviluppo pre-marketing. 


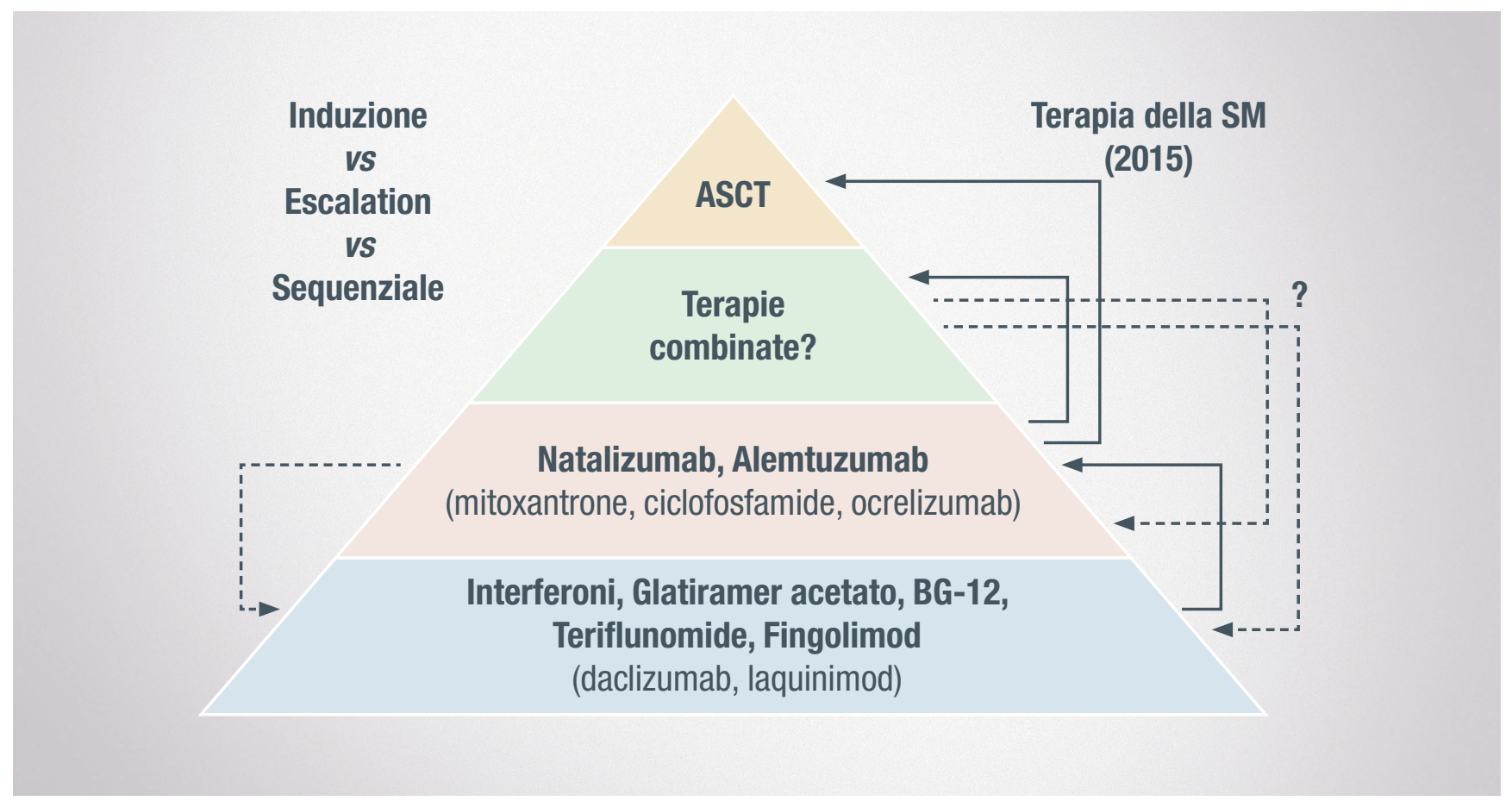

Figura 2. Selezione delle strategie terapeutiche per la terapia della sclerosi multipla (SM). ASCT, trapianto autologo di cellule staminali; BG-12, dimetilfumarato.

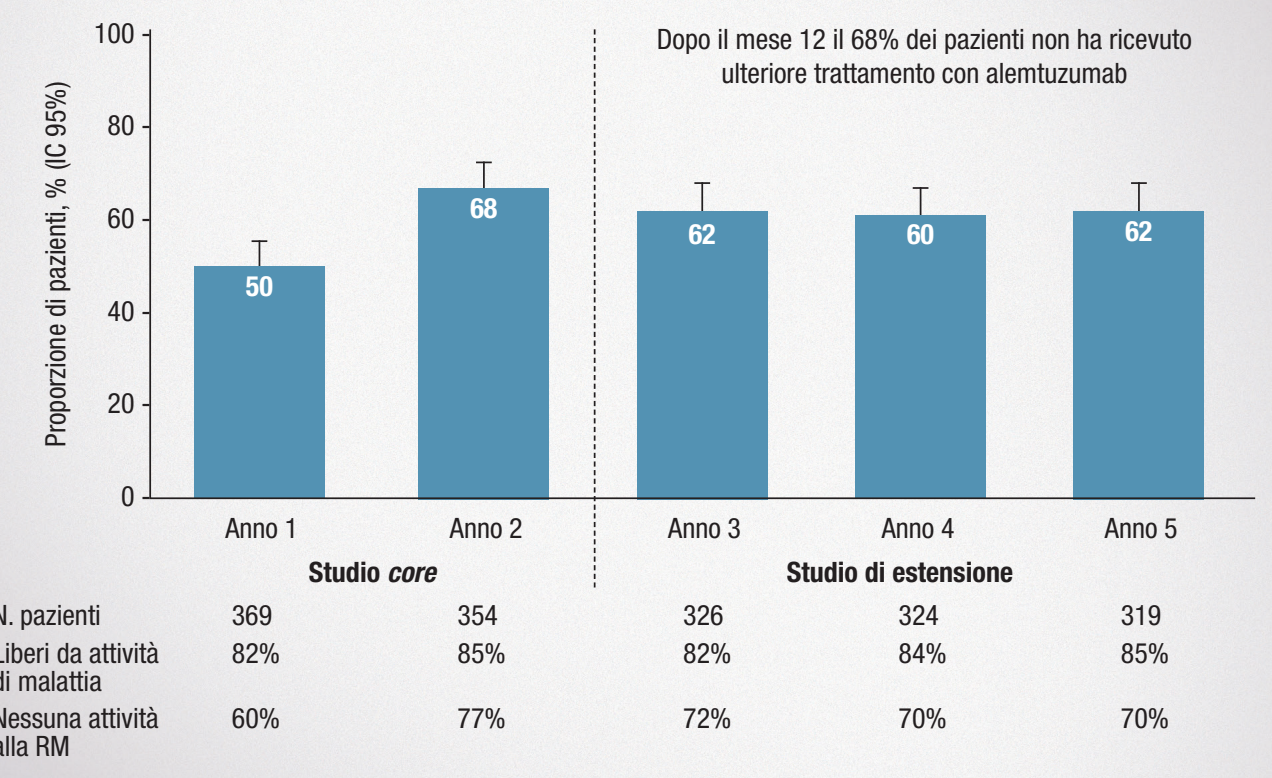

Figura 3. Proporzione di pazienti trattati con alemtuzumab che hanno raggiunto e mantenuto lo stato NEDA (no evidence of disease activity) fino a 5 anni (modificata da [2]). 


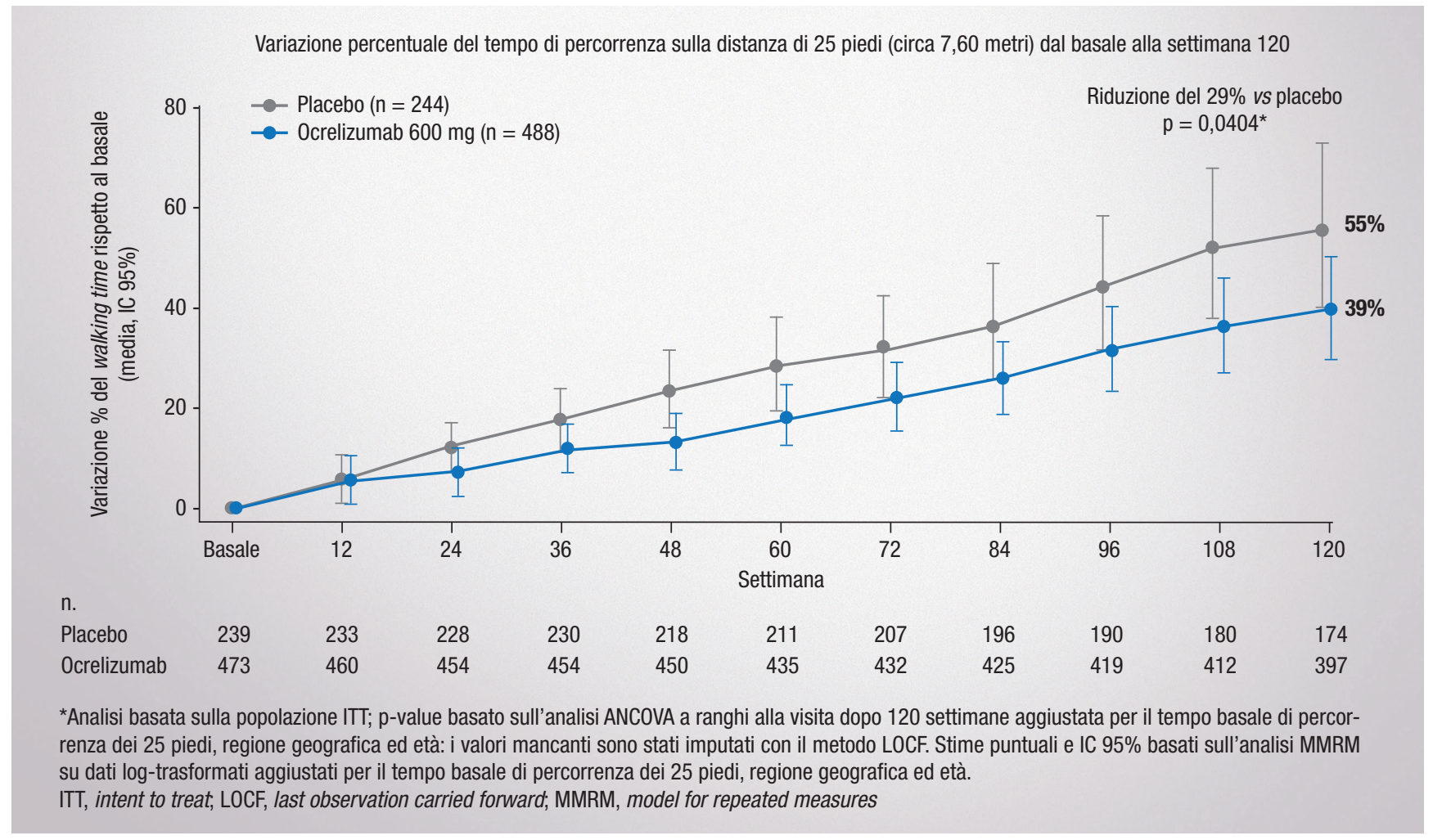

Figura 4. Riduzione significativa della velocità di progressione del tempo di percorrenza (walking time) (modificata da [3]).

Un settore in cui la carenza di risposta terapeutica è invece enorme è quello delle forme progressive (sia primarie che secondarie). Fino a poco tempo fa nessuna opzione terapeutica era disponibile per arrestare la lenta degenerazione assonale tipica di queste forme. Recentemente un anticorpo monoclonale anti-CD20 (ocrelizumab) ha dimostrato i primi incoraggianti risultati, sia sul versante clinico che su quello radiologico [3] (Figura 4).

Auspicabilmente oggi è possibile ipotizzare l'arresto dei processi degenerativi tipici della SM (demielinizzazione e, ancora in misura minore, apoptosi). Rimane tutto da esplorare, invece, il campo della rigenerazione del tessuto lesionato, una problematica che la SM condivide con gran parte delle malattie del SNC (ictus, trauma, tumori, proteinopatie ecc.). Le notevoli aspettative poste in nuovi e innovativi approcci (es. ripresa della mielinizzazione mediante anticorpi anti-LINGO) per le patologie demielinizzanti iperacute (neurite ottica) e subacute-recidivanti (SM attiva) aspettano l'esito degli studi di fase II i cui risultati dovrebbero rendersi disponibili nel corso del 2016. Un ultimo aspetto che non trova risposta adeguata è il trattamento della complicanza più temuta dai pazienti in corso di trattamento immunomodulante intensivo: la leucoencefalopatia multifocale progressiva (PML). Per tale infezione da virus JC al momento non esistono terapie efficaci, se non la semplice sospensione del trattamento immunologico nella speranza di una immuno-ricostituzione efficace in tempo per impedire lo svilupparsi di danni strutturali al parenchima cerebrale per effetto diretto del virus.

\section{I bisogni socio-assistenziali dei pazienti}

\section{Organizzazione}

Le problematiche socio-assistenziali costituiscono spesso il motivo di principale sofferenza personale di una notevole quota di pazienti affetti da SM. Un recente stu- 
dio della SDA Bocconi in collaborazione con l'Azienda Ospedaliero-Universitaria di Ferrara ha chiaramente dimostrato come il consumo di prestazioni relative alle terapie specifiche per la SM - le DMT - si concentri nel gruppo di pazienti a bassa disabilità e si riduca gradualmente all'aumentare della disabilità fino a scomparire in presenza di disabilità elevata. Questa riduzione del consumo di terapie è controbilanciata dal crescente ricorso a prestazioni non specialistiche per la SM (ad esempio, visite non neurologiche) e ai ricoveri, sia nelle U.U.O.O. di Neurologia sia in quelle di Medicina Generale, Riabilitazione, Gastroenterologia, Lungodegenza e Neuroriabilitazione [4]. I pazienti con disabilità assente o iniziale incidono maggiormente sulla spesa farmaceutica e presentano una buona aderenza, favorita dalla ridotta fragilità, mentre i pazienti con malattia più avanzata consumano maggiormente prestazioni diagnostiche strumentali e di laboratorio e presentano tassi di ospedalizzazione molto più elevati. Storicamente la Neurologia rappresenta l'U.O. che tradizionalmente è in grado di gestire queste situazioni iniziali. All'evolvere della patologia, però, e in condizioni di fragilità socio-ambientale, aumenta la richiesta d'integrazione con la rete di servizi territoriali e con professionalità diverse dalla neurologia, in modo da garantire la continuità della presa in carico, sia a livello di singola Azienda Sanitaria che in sede interaziendale. Tra i vari strumenti di controllo gestionale va assumendo un ruolo sempre più centrale la creazione di Registri di malattia, su base sia nazionale sia regionale, che consentirebbero di:

1. Avere un quadro molto preciso del numero di pazienti, della loro distribuzione geografica (d'origine e di residenza), che consenta analisi epidemiologiche e ambientali anche attraverso incroci dei database dei vari Centri SM regionali.

2. Monitorare e controllare la spesa sanitaria, specialmente quella farmacologica (molto alta per questa patologia).

3. Disporre di uno strumento di archiviazione e certificazione standardizzato.

La via attualmente più seguita per migliorare il percorso assistenziale dei pazienti dalla diagnosi all'accesso alle terapie e alla riabilitazione è la creazione di un Percorso Diagnostico-Terapeutico Assistenziale
(PDTA) specifico per la SM. La formulazione nelle varie realtà regionali di PDTA strutturati che consentano di coordinare tutti i Centri SM in un'unica rete assistenziale, garantisce una qualità omogenea sia delle scelte mediche che della presa in carico delle persone affette da SM nell'ambito di un "percorso", non come una sommatoria casuale di una serie di singole prestazioni diagnostico-terapeutico-assistenziali. Il primo PDTA italiano interamente dedicato alla SM, redatto dalla Regione Siciliana (Supp. Ord GURS del 24-10-2014), che si proponeva di "prendere in esame e mettere a fuoco un percorso diagnostico, terapeutico e assistenziale specificatamente dedicato alle persone con SM, che metta concretamente la persona al centro" (...), valutandone "l'impatto sociale rispetto alla complessità di questa malattia ed alle difficoltà crescenti che l'offerta attuale da parte del Servizio Sanitario Nazionale determina in termini di oneri assistenziali a carico delle famiglie nelle quali è presente una persona con malattia cronica ad andamento evolutivo", è stato seguito da quello di numerose altre Regioni italiane (Emilia Romagna, Veneto, Piemonte, Puglia ecc.) che nel loro insieme costituiscono, pur con le diversificazioni legate all'inserimento nei rispettivi Sistemi Sanitari Regionali (SSR), un corpus normativo di riferimento essenziale per la gestione pubblica integrata di questa patologia.

\section{Informazione}

Per i pazienti affetti da SM è ormai fondamentale l'informazione e la comunicazione, la tutela dei diritti e la personalizzazione delle cure. In particolare i pazienti, sempre più orientati a reperire informazioni sui farmaci o sulle procedure esistenti o in sviluppo per la loro malattia sulla rete web (es. www.aism.it, www.nationalmssociety.org, www.ectrims-congress, eu, ecc.) e meno a usufruire del consiglio del neurologo del Centro SM o del medico di medicina generale (MMG), sono spesso confusi dalla gran messe di dati che non riescono a gestire per carenza di cultura medica o semplicemente perché troppo coinvolti dalla loro malattia per mantenere un adeguato giudizio critico. In tale senso è cruciale l'accesso a referenti 
specializzati presso i Centri SM a loro dedicati, ma le carenze organizzative e finanziarie di tali strutture (tuttora quasi mai sostenute da quote di finanziamento specificamente previste da parte dei vari SSR) impediscono la rapida comunicazione bidirezionale con i loro assistiti. Sempre più spesso, infatti, i pazienti segnalano difficoltà ad accedere alle informazioni sull'organizzazione dei vari Centri, dei loro servizi e delle figure di riferimento. Ancora più infrequente è la stipula di accordi procedurali per agevolare la $\mathrm{CO}-$ municazione e il passaggio della presa in carico tra medici dei Centri SM ospedalieri o territoriali e MMG. Nonostante da vari lustri in Italia sia attiva una cartella informatizzata che consente di conservare e "posizionare" dati clinici, radiologici, biologici ed eventi biografici di qualsiasi paziente con SM (cartella iMed), solo da poco sono in corso tentativi di renderla obbligatoria per i Centri delle reti regionali in modo da poter studiare i flussi di pazienti nell'ambito dell'intera rete, la risposta e la somministrazione sequenziale dei vari farmaci nella moderna storia naturale della malattia (quella, cioè, modificata dai vari DMT); ciò permetterebbe anche di utilizzare questa rilevante mole di dati per dare una risposta a vari quesiti scientifici possibile solo dalle analisi post-marketing mediante, per esempio, l'uso di metodologie statistiche come il propensity score [5]. Ridotto è infine in Italia il numero degli operatori socio-sanitari invece necessari per sostenere i pazienti nei loro problemi di motilità.

Il margine futuro di sviluppo in quest'ambito sarà la copertura totale del territorio italiano con l'adozione di PDTA in tutte le regioni d'Italia e l'integrazione di tutti questi algoritmi gestionali in un unico documento che possa regolamentare con le stesse regole la gestione clinica, assistenziale ed economica di tutti i pazienti italiani.

\section{Percorso personalizzato}

Il passaggio dalla rete per l'acuzie a quella per la cronicità (la cosiddetta "presa in carico" dei pazienti) viene ormai considerato una delle tappe del percorso personalizzato dei pazienti affetti da SM, dalla diagnosi alla terapia. La presa in carico utilizza servizi differenti a seconda del grado di disabilità, del livello personale di coinvolgimento sociale o familiare e della presenza di forme di disturbo psichico o cognitivo. Per i pazienti più fragili sotto il profilo socio-ambientale, spesso il ricorso all'ospedale sembra supplire all'assenza o carenza di cure intermedie o domiciliari. L'assistenza domiciliare integrata (ADI), infatti, è utilizzata dai pazienti affetti da SM lieve in percentuali irrisorie (1-5\% nelle varie regioni), mentre si registrano livelli più alti sempre nei pazienti con livelli di disabilità e fragilità elevata (circa il 40\%). Il ricovero ordinario è, invece, attivato per gestire le fasi acute all'esordio della patologia e le complicanze che insorgono con gli stadi di aggravamento della disabilità. La Neurologia rappresenta, come dianzi riportato, l'Unità Operativa che gestisce questi livelli assistenziali. All'evolvere della patologia e, soprattutto, delle condizioni di fragilità socio-ambientale, si registra invece un bisogno che supera i confini della singola U.O. e richiede una capacità di integrazione con la rete di servizi territoriali e con professionalità diverse dalla Neurologia, sia per coprire lo spettro di bisogni sanitari e assistenziali sia per garantire la continuità della presa in carico, a livello aziendale e interaziendale.

\section{Dignità dei malati}

L'implementazione di una rete integrata di servizi regionali facilita l'umanizzazione dell'assistenza per tale malattia. Occorre saper ascoltare i pazienti per elevare la qualità delle cure e garantire la loro dignità di esseri umani. A fronte di sempre più marcate carenze di risorse, medici e infermieri continuano a prendersi cura delle persone, oltre che a curarle. I centri SM trattano una malattia cronica che richiede competenze altamente specialistiche per le quali non si può fare a meno dell'ospedale, ma occorre realizzare reti sul territorio soprattutto per quanto riguarda il rapporto col MMG, i centri di riabilitazione e i centri socio-sanitari, in modo da condividere competenze e servizi.

\section{Presa in carico globale e continuità ospedale-territorio}

La complessità e la varietà dei quadri clinici che caratterizzano la SM determinano la necessità di una 


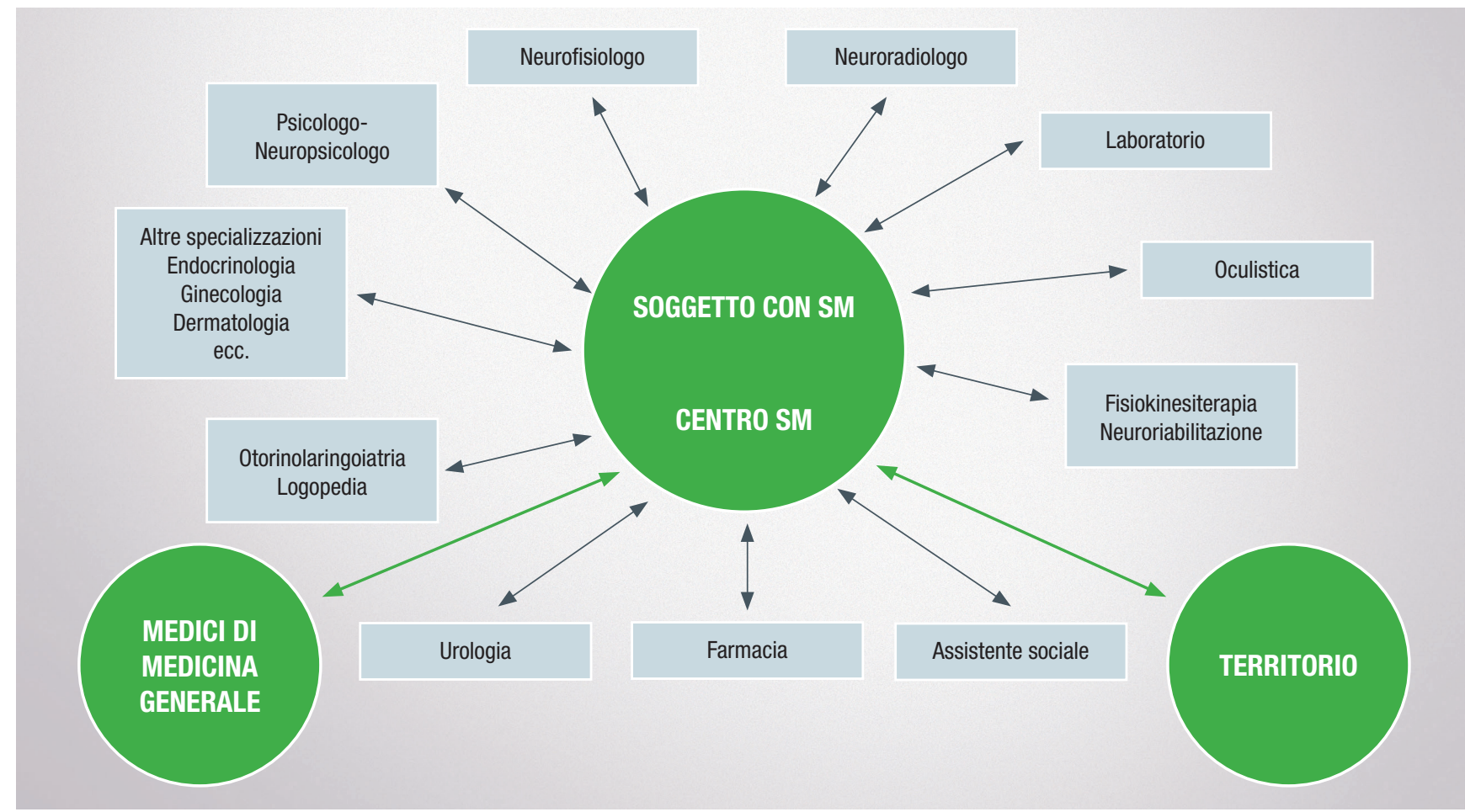

Figura 5. Rete multidisciplinare di servizi ospedalieri e territoriali per la presa in carico del paziente con sclerosi multipla (SM).

presa in carico globale della persona con SM, con interventi da parte di una rete di servizi che coinvolgano operatori sanitari e sociali, composta da vari specialisti che intervengono in tempi e luoghi diversi dal momento della diagnosi e per tutto il percorso terapeutico e assistenziale, mantenendo sempre il carattere di approccio terapeutico interdisciplinare inserito in un programma unico definito e condiviso.

La rete assistenziale per le persone con SM è formata dai referenti di Servizi diversi: Centro di riferimento SM, Servizio riabilitativo, Azienda servizi alla persona (ASP), MMG/pediatra di libera scelta (PLS), Rete specialistica territoriale, Servizio sociale territoriale, Centri per l'impiego. Per i pazienti con livelli di fragilità socio-ambientale elevata è necessaria una presa in carico ancora più onnicomprensiva, in grado di garantire continuità e appropriatezza, sia preventiva che di sorveglianza sociale, coinvolgendo attivamente numerose figure professionali, quasi sempre raggruppate nell'ambito di un Centro
$\mathrm{SM}$; tra di esse non possono mancare l'assistente sociale, lo psicologo o psichiatra e l'infermiere specializzato nella SM (Figura 5).

Alla presa in carico del paziente tale team predispone un piano individualizzato onnicomprensivo, teso a soddisfare i bisogni sanitari e socio-sanitari della persona e la sua garanzia sociale mediante servizi e interventi differenziati, tra loro integrati, che debbono adeguarsi all'evolversi dei bisogni. La persona deve avere un punto di riferimento certo a livello territoriale, che coordini l'intero percorso di presa in carico come ad esempio un "case manager", sia esso un medico o un infermiere specialista di SM, che dovrà "coordinare l'intero percorso assistenziale, seguire direttamente il paziente nei diversi contesti coordinando le informazioni sugli aspetti clinici e sociali rendendole disponibili a tutti gli operatori coinvolti, nonché contribuire a migliorare la sua qualità di vita rilevandone e sviluppandone le capacità residue al fine di aumentarne l'autonomia ed evidenziandone i bisogni secondo un ordine di priorità". Una presa in carico globale deve poter assicurare 
"un'assistenza che risponda a criteri di qualità e competenza; una presa in carico globale del paziente con continuità assistenziale in ogni fase di malattia; un sostegno reale alle famiglie per quanto riguarda gli aspetti gestionali e amministrativi".

Il raccordo tra la rete sanitaria e i servizi sociali territoriali, oltre alla pianificazione delle attività socio-assistenziali, è garantito dal coinvolgimento dell'assistente sociale del Comune e/o dal coinvolgimento del "case manager" insieme ai referenti dei servizi presenti a livello territoriale.

Nelle fasi più avanzate di malattia diviene essenziale la figura del caregiver: generalmente esso è un familiare, un convivente o una persona specificamente incaricata che svolge una funzione di assistenza diretta. La sua individuazione precoce lungo la fase di peggioramento della malattia è essenziale nella realizzazione del percorso assistenziale integrato. È necessario strutturare un percorso di formazione specifico per queste figure che ne adegui le competenze alle specifiche necessità del paziente e le integri con la rete assistenziale disponibile.

Per quanto riguarda l'integrazione ospedale-territorio, il Piano Sanitario Regionale sancisce che il "principio della continuità delle cure ha un ruolo centrale in tutti i disegni organizzativi ed assistenziali nella gestione delle cronicità e deve favorire, garantendo il massimo livello di confronto specialistico tra le diverse strutture ospedaliere e fra ospedale e territorio, la razionalizzazione delle risorse disponibili, la generazione di cultura, lo sviluppo di sistemi informatici interattivi, idonei sistemi informativi e adeguati sistemi di verifica della qualità, permettendo così l'elaborazione di linee guida di buona pratica clinica ed assistenziale e la diffusione di programmi di formazione in relazione ai bisogni emersi".

\section{Conclusioni}

Numerosi progressi sono stati recentemente compiuti nel trattamento e nella gestione multidimensionale dei pazienti affetti da SM. Rimangono insolute alcune importanti criticità terapeutiche per pazienti con forme cliniche particolarmente resistenti all'osservazione scientifica e alla sperimentazione farmacologica. L'implementazione della rete dei Centri SM sul territorio nazionale in modo articolato sulla base delle caratteristiche dei vari SSR e con il supporto di "manuali d'istruzione" come i vari PDTA, la creazione dei Registri di malattia, il sempre maggiore utilizzo dei supporti informatici di registrazione dei dati clinici e l'integrazione tra strutture sanitarie e assistenziali, rappresentano punti salienti di un chiaro miglioramento della gestione olistica di questa malattia. A fronte di questa visione ampiamente positiva, un segnale controcorrente viene dall'incombente riduzione delle risorse economiche e di personale che affligge il nostro Sistema Sanitario Nazionale (SSN), in sintonia con quanto sta purtroppo avvenendo in tutti i Paesi occidentali. È auspicabile che tali congiunture negative non arrestino il trend positivo che la ricerca biomedica e l'aumentata sensibilità politico-assistenziale sta regalando ai soggetti affetti da SM.

\section{Disclosures}

Conflicts of interest: The author declares he has no conflict of interests related to the article.

\section{Bibliografia}

1. Giovannoni G, Turner B, Gnanapavan S, et al. Is it time to target no evident disease activity (NEDA) in multiple sclerosis? Mult Scler Relat Disord. 2015;4(4):329-33.

2. Havrdova E, Arnold DL, Cohen JA, et al., on behalf of CARE-MS I Investigators. Durable efficacy of alemtuzumab on clinical outcomes over 5 years in treatment-naive patients with active relapsing-remitting multiple sclerosis with most patients not receiving treatment for 4 years: CARE-MS I Extension Study. Presented at $31^{\text {st }}$ ECTRIMS Congress. Barcelona, Spain, 7-10 October, 2015

3. Montalban X, Hemmer B, Rammohan K, et al., on behalf of the ORATORIO clinical investigators. Efficacy and safety of ocrelizumab in primary progressive multiple sclerosis - results of the phase III, double-blind, placebo-controlled ORATORIO study. Presented at $31^{\text {st }}$ ECTRIMS Congress. Barcelona, Spain, 7-10 October, 2015.

4. Pacileo G e il Multiple Sclerosis Management Lab. La fragilità socio-ambientale dei pazienti SM: modelli integrati ospedale-territorio per la SM. Corso SDA Bocconi, 31.10.2014.

5. Trojano M, Pellegrini F, Paolicelli D, et al. Observational studies: propensity score analysis of non-randomized data. Int MS J. 2009; 16(3):90-7 